\section{"Managing" Interdisciplinary Materials Research at a University: Memoirs of an MRL Director H.K. Birnbaum}

The virtues of interdisciplinarity are more often praised than practiced. The reasons are not difficult to identify. People from different disciplines must integrate a bit, loosening their individual departmental or disciplinary identities, and be willing to share their knowledge, facilities, and resources for commonly perceived objectives. Materials research is a wonderful example for interdisciplinarity. The tent is large and promising and beckons scientists and engineers from practically all areas of knowledge. MRS Bulletin represents this very quest. Despite the obvious attractions, universities and research laboratories have not been very successful in building truly interdisciplinary institutions. Many university departments have materials research only in name, but remain predominantly metallurgy- , chemistry- , chemical engineering- , or ceramics-oriented, the disciplines from where the new departments came into being, and the synergy that a true integration would unleash is absent. There are only a few examples of institutions where the pooling of knowledge and resources have succeeded and many are in the United States. This may not indeed be accidental as the United States still thrives by experimenting and institutions are also not that old to become the guardians of tradition. The University of Illinois is an example where materials research is perceived and practiced as a truly integrated discipline. In the following article, H.K. Birnbaum, who was director of the Frederick Seitz Materials Research Laboratory for over a decade, describes his experience in heading this institution.

V.S. ARUNACHALAM

The field of materials science has emerged from its beginnings in the mid1950s to become a large, complex interdisciplinary research and educational endeavor involving efforts of scientists 1960s through the efforts of people such as John von Neumann, Fred Seitz, Harvey Brooks, Charles Yost, and Don Stevens. They formed a group of university and government leaders who had the vision of bringing the fields of physics, chemistry, metallurgy, and ceramics together into an interactive effort to understand the basic sciences of materials. ${ }^{1}$ These IDLs-later termed Materials Research Laboratories (MRLs) and Materials Research Science and Engineering Centers (MRSECs)have continued to develop under the sponsorship of the National Science Foundation (NSF) and the Department of Energy (DOE), and have spread to include the efforts of a large number of groups at universities around the country, and in various forms have been developed overseas (see Sidebar). Having managed the large interdisciplinary materials research effort at the University of Illinois Frederick Seitz Materials Research Laboratory for 13 years, I thought it might be useful to record some of my observations and thoughts concerning this endeavor on the occasion of my stepping down as director. These views have been developed to apply to a large, continuing interdisciplinary effort rather than the more limited single Interdisciplinary Research Group MRSECs currently funded by the NSF. I believe they have general relevance to interdisciplinary efforts regardless of size.

In managing these laboratories it is important to recognize that even in these days of "team" research, great ideas and concepts are almost always the product of individuals or small closely interactive groups of researchers. While group efforts may be appropriate in the development of a research effort (particularly in applied research), research conceptualization is nurtured by discussions and interactions, but is produced by individuals or very small groups of closely interacting individuals. These interactions are 
almost impossible to plan, and occur rarely and unpredictably. Within an interdisciplinary research organization, we can expect that productive scientists will often interact in small groups, the composition of which will change as the participants seek out those who meet their creative needs. It is counterproductive to try to impose a structure on these efforts-particularly within the scope of what is generally termed "basic" research. In recent years we have seen examples of exciting science that have resulted from the creativity of individuals and that have been quickly followed by the funding of large group efforts. One example is the discovery in 1986 of high- $\mathrm{T}_{\mathrm{c}}$ superconductivity in a class of materials in which transport properties were long neglected. This discovery by Alex Müller and Georg Bednorz was followed by large coordinated efforts worldwide. These group efforts resulted in a steady progression in our understanding of the phenomena and in some potential technological applications, but in no great advances. These await new, imaginative ideas that will most likely come from individual scientists.

A director of an interdisciplinary research organization can facilitate productive interactions between creative individuals by making the interests and capabilities of the community members known to each other and by removing any obstacles to the interactions. It is very helpful to have the researchers housed in contiguous space designed to encourage informal discussion and interactions as well as to sponsor presentations of concepts in their early stages of development. In the context of a university, this is difficult to achieve as there are countervailing forces drawing faculty to their disciplinary, departmental buildings. The interdisciplinary MRL needs to offer high quality space for offices and laboratories, for both students and faculty - even at the cost of offering faculty a second office. Cooperation of the departments is also required, although they would rather have their faculty closely associated with the department. The sponsoring of social events at which discussions can occur is money well spent. These events can be as simple as a daily coffee at which students and faculty are encouraged to attendcookies work well to attract students-or occasional dinners. My observation is that at these events, the conversation drifts toward science. The ability to develop these events requires that discretionary funds be available to the MRL director as it is difficult to fund these events from contract funds.

\section{It is highly desirable that funding agencies allow flexi- bility in the expenditure of research funds, followed by post-facto justification based on the quality of the science.}

An issue faced by MRLs (often in the justification of their research support) is validation of their interdisciplinary interactions. There is no generally accepted metric for these interactions and they are best judged in retrospect. Joint publications, artificial groupings, and the like do not provide a satisfactory way of determining useful interactions. Most creative interactions are informal and do not result in objects that can be counted. Despite the difficulty in making this measurement, MRLs are requested to demonstrate that their members work in an interdisciplinary manner, and since it is easier for program directors and reviewers to count than to make judgments of quality, there is pressure to generate items that can be counted. Measures such as joint publications are useful but do not reflect the discussions and exchanges of ideas that often underlie great research. An illustrative example is the development of a method for detwinning single crystals of the YBCO superconductor that was based in part on an informal discussion, at an MRL coffee, of a way of detwinning hydride single crystals. ${ }^{2}$

Flexibility in research funding is essential to the development of imaginative, excellent research programs. Such flexibility is an absolute requirement to allow development of novel ideas that have not been anticipated at the time the proposal for funding was written. This flexibility must include both changes in the amount of funding support for any particular effort, the direction of research, and the ability to add new researchers to the interdisciplinary effort. As recent examples where this flexibility was necessary I

\section{Interdisciplinary Materials Research Laboratories}

While the Illinois Frederick Seitz Materials Research Laboratory currently receives its major support from the Department of Energy Office of Science, it has in the past received significant support for its interdisciplinary programs from the National Science Foundation (NSF), and before that from the Defense Advanced Research Projects Agency. The NSF is currently the major source of funding for universitybased interdisciplinary funding in materials science (see its web site at http://www.nsf.gov/mps/dmr/mrsec.htm).

Currently multi-focus Materials Research Science and Engineering Centers (MRSECs) are supported at the California Institute of Technology, University of California-Santa Barbara, University of Chicago, Cornell University, Harvard University, University of Maryland-College Park, Northwestern University, University of Pennsylvania, Princeton University, and the University of WisconsinMadison.

In addition, single focus interdisciplinary MRSECs are funded at the University of Alabama, Arizona State University, Brown University, Carnegie Mellon University, University of Colorado-Boulder, Columbia University, University of Houston, Johns Hopkins University, University of Kentucky, University of Massachusetts-Amherst, Michigan State University, University of Minnesota, University of Oklahoma, Pennsylvania State University, Stanford University, SUNY at Stonybrook, SUNY/ Polytechnic University/CUNY, and the University of Virginia.

While all of these NSF-supported programs are specified as "interdisciplinary," the research supported is in effect circumscribed by the initial proposal. See the NSF web site already provided for a specification of the areas of research in each MRSEC. Support of comprehensive Central Instrumentation Facilities is generally possible only at the funding levels of the largest multi-focus MRSECs.

In addition to the formally supported programs, many universities in the United States and elsewhere have used their own resources and "pooled" individual grants to form interdisciplinary materials research programs and shared Central Facilities. A very incomplete list includes Ohio State University, Washington State University, Arizona State University, University of Florida, Oregon State University, McMaster University (Canada), and many others. The validation of the concept pioneered by Fred Seitz, John von Neumann, Harvey Brooks, Charles Yost, and Don Stevens in the 1950s and early 1960s lies in the fact that these interdisciplinary efforts are at the heart of modern materials research and education at universities. 
can cite the periods following the announcement of "high-temperature superconductivity" and of "cold fusion." In both cases we were able to allow MRL researchers to use their existing funding to quickly explore their "best" ideas. In the first instance this allowed development of excellent science that led to independent funding, while in the latter case it allowed a potentially exciting phenomena to be shown to be a chimera. Unfortunately, this flexibility is increasingly difficult to maintain in light of funding agency requirements for "accountability" and the tendency for review panels to want research programs to be completely defined. It is highly desirable that funding agencies allow flexibility in the expenditure of research funds, followed by post-facto justification based on the quality of the science. This requires a significant level of trust between the funding agencies and the MRL director.

The "other side of the coin" is that the MRL, by whatever process it uses, must be willing to terminate less productive programs to obtain the funds for redirecting support to the most promising efforts. In the case of the Illinois MRL, this amounted to an annual 5\%-10\% "turnover" of program support. The choice of which programs to include within an MRL is perhaps the most difficult decision made by this organization. This decision will affect colleagues with whom the director continues to interact on a frequent basis in many areas of academic life. The MRL director must ensure that these decisions are made in a manner that utilizes the best intellectual judgments available within and outside the organization. It is critical that in making these judgments the process utilize reviewers outside the MRL and outside the university that contains the MRL. Changes in the program must be carried out interactively with the funding agency, and must take into account the results of the periodic reviews carried out by the funding agencies. Additionally the judgments, which often strain "collegiality," must be "fair" and be perceived as "fair." In the context of a university, having faculty salaries paid by the university rather than being derived from MRL funds enhances the flexibility of these program decisions. The related issue of enabling students to complete their theses when the program is terminated is often a difficult one and requires cooperation by the student's home department. At Illinois the departments receive some discretionary funds from the MRL activities and hence they should be willing to assist in the support of students whose

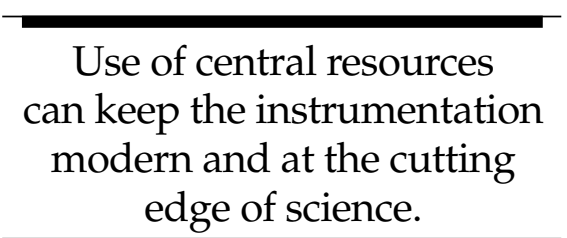

advisors' programs are being phased out by the MRL.

Cost sharing is now required in most large Federally supported interdisciplinary programs, and generally the university must assist with this as non-Federal funds are required. More important than the dollar commitment by the university is the "intellectual" commitment to the activities of the interdisciplinary program. This "intellectual" commitment should include the realization that support (financial and other) may be required to maintain the interdisciplinary effort after the Federal grant expires, if and only if the activity remains intellectually vital to the university. It is important that the university "buy into" the MRL effort in ways that allow the MRL director to maintain the vitality of the program. In many cases this means a willingness to provide "discretionary" funds to allow the initiation of new efforts. In the case of the Illinois MRL, the university has developed a "seed funding" program to start research programs that in a number of cases have eventually received significant Federal funding. The university (often using state-funded programs) has also provided significant support for central instrumentation facilities at times when Federal funds were removed, has supplemented Federal funding for longrange research efforts (e.g., the Illinois Superconductivity Center), and has supported major facility development (e.g., the development of $\mathrm{x}$-ray beam lines at the Advanced Photon Source). Since university administrations are subjected to many demands, maintenance of their focus on the needs of the MRL has not been an easy task, but it is an essential one. In the case of the Illinois MRL, a particularly important source of these non-Federal funds is the award to the MRL of discretionary funds in proportion to the overhead charges on grants obtained by the MRL. The flexibility provided by these funds is extremely important for starting new research efforts, cost sharing on Federal grants, and supporting shared instrumentation facilities that are provided on a continuing basis with few limitations on their use.

All of this support from the university requires clear and workable channels of interaction with department heads and the university administration. This is particularly important because the MRL director interacts with several departments that reside in different colleges. The nature of these interactions will vary with the university culture. At Illinois the MRL Director reports to the Dean of Engineering and has the status of a department head, although the MRL cannot hire faculty. In other universities, the reporting line is to the Provost. Whatever the arrangement, the director must have a degree of independence from department heads, as there is only partial overlap between the interests of the MRL and the departments. A large measure of cooperation with the departments is required, in the hiring of faculty, on which the MRL depends. The needs of the Illinois MRL were often factored into faculty hiring decisions, and the MRL often assisted with the needed "recruitment packages." While development of the MRL research efforts require faculty with particular research interests, these needs cannot be the basis on which hiring decisions are made. Regardless of the needs of the interdisciplinary program and possible opportunities, the MRL should resist the temptation to hire tenure line faculty, even if the opportunity to do so is offered. The MRL should limit itself to adding professional staff and research associates.

A good deal of my time as MRL director was spent in maintaining support from the broad university community and in arranging for significant input from all levels of the university. While this process could be formalized by the use of committees, I preferred to do this less formally by spending significant time with all groups involved with the MRL. The dependence of the MRL on Federal agency funding mandates the maintenance of excellent relations between the MRL director and the agency personnelan activity that requires considerable expenditure of time and energy and that requires sensitivity to the changing needs and demands of the supporting agencies.

Formation of an interdisciplinary laboratory provides a major opportunity for the effective use of capital equipment investments. About 1980, the Illinois MRL decided to centralize all of the major instrumentation into a series of central facilities, "owned" not by individual faculty, but by the MRL community. With the assistance of the departments and the university for cost sharing, and of faculty in the writing of proposals for major instrumentation, the MRL used a significant fraction of its Federal and discretionary funding to develop an excellent base of advanced instrumentation for materials research. These facilities could otherwise not have been afforded and the capabili- 
ties they offered benefited both the MRL community and the wider University of Illinois and U.S. research communities. University departments used the existence of these facilities as a recruiting attraction both for new faculty and for graduate students. While the existence of these central facilities was almost unique to the Illinois MRL at their inception, the value of this concept has been widely recognized and is frequently emulated at other institutions.

At the Illinois MRL, the staff of the central facilities serve a number of important roles. Central facility staff maintain the instrumentation at a very high level of operational capability, develop new capabilities for the instruments and new analysis methods, instruct the users in the operation of the instruments, and generally facilitate the research endeavor. A major function is the education of graduate student and research associate users in instrumentation science and in the practical operation of the instruments. While the staff is encouraged to enter into cooperative research efforts with the users, they generally do not carry out measurements for the users. Consistent with the teaching responsibilities of the MRL, users are generally expected to perform their own measurements, with the staff providing assistance and helping with the interpretation of the data, if necessary. For some instruments, there is much more intense involvement of the staff with the usersparticularly in the early stages of instrument and analysis development. A consequence of this mode of operation is that the MRL annually educates a large number of users in instrumentation science (about 300 per year), and thus contributes to the educational mission of the university in a very direct manner. A recent effort of the central facilities is to develop remote operation of the instruments over the Internet. This allows users to operate the instruments from remote locations and, perhaps more importantly, to view the output of the instruments and to interact with the person at the instrument from a remote location in real time. These capabilities both facilitate research use of the instruments and their use in education of undergraduates who do not currently use the central facilities. These capabilities have not yet been fully utilized, but they offer great future potential. The high quality of the central facility staff is critical to the success of these efforts, and the ability to attract and support these individuals requires the centralized funding and longterm stability of an MRL structure. Since the professionals working in the central facilities do not have faculty positions, the "care and feeding" of these professionals, who are often as qualified as faculty, requires great attention by the MRL director considering the "pecking order" that prevails at universities.

Access to the central facility instrumentation is on an open and equal basis within the MRL, the University of Illinois, and the U.S. research community. Only in the cases where time constraints are severe are priority access arrangements made. Central facility staff assists in arrangements for non-local users, and often works closely with them to facilitate obtaining results from their instrumentation time. Once instruction in the use of the instrument is completed and the user certified for the operation of the instrument, it is available 24 hours per day, seven days per week, on a "sign-up" basis.

The experience at the Illinois MRL is that major advantages accrue from the presence of these central facilities:

- Researchers have access to a wide range of instrumentation without needing to be expert in the technique as they are taught and assisted by expert staff. Such access is uninhibited by issues other than competency.

- Access to a range of instrumentation allows users to select a suite of techniques that are most suited to their research needs. The staff often assists in the choice of the most suitable techniques.

- Use of central resources can keep the instrumentation modern and at the cutting edge of science.

- Centralized management allows the phasing out of facilities that no longer serve the purpose of the research program.
- Centralization of the facilities within the MRL building allows optimal use of staff, particularly for maintenance, and allows "intellectual cross-fertilization" between the users and staff focused on various techniques.

- Use of the central facilities greatly promotes interdisciplinarity in the research endeavor as these serve as places where researchers from different programs meet, discuss science, and interact.

- Use of the central facilities by those parts of the university that cannot be directly supported by the MRL is a strong basis for general university support.

These comments and observations reflect my involvement with the University of Illinois MRL since its inception in 1963 and my experiences in trying to manage its research program for the past 13 years. I have indicated some of the things that have worked, ignored those that have not, and glossed over many details and the vigorous discussions that led to the current arrangement. While I believe that there is generality in the above comments, each institution must reach its own best arrangement-and knowing educational institutions, they will all differ. For materials science, and probably for many other fields of intellectual endeavor, what is described briefly here has been, and continues to be, a useful route to enhance scholarly endeavor.

\section{References}

1. Frederick Seitz, On the Frontier, My Life in Science, (AIP Press, New York, 1994), p. 140.

2. J. Giapintazakis, D.M. Ginsberg and P.D. Han, J. Low Temperature Phys. 77 (1989) p. 155.

H.K. Birnbaum is an emeritus professor of materials science and engineering at the University of Illinois at Urbana-Champaign where he served as the director of the Frederick Seitz Materials Research Laboratory from 1986 to 1999.

Material Matters is a forum for expressing personal points of view on issues of interest to the materials community.

\section{MRS Web Site exclusive} WWW-mrs.org

Materials Musings: "Curiouser and Curiouser," Prof. Robert W. Cahn (Cambridge University) Access this series of essays and articles exploring various facets of the world of materials research. 